\title{
Psicooncología
}

ISSN: 1696-7240

\section{Información sobre la quimioterapia paliativa en pacientes con cáncer avanzado: una revisión}

\author{
José Miguel Navarro Jiménez ${ }^{1}$; Juan Antonio Cruzado²; María Jesús Boya Cristia ${ }^{3}$
}

Recibido: 28 de noviembre de 2016 / Aceptado: 14 de abril de 2017

Resumen. Introducción. La quimioterapia paliativa es el tratamiento específico de primera elección en la mayoría de los pacientes oncológicos con enfermedad avanzada y es esencial que los pacientes entiendan los objetivos del tratamiento y las expectativas se ajusten al proceso de la enfermedad.

Objetivo: Hacer una revisión sistemática sobre las creencias y expectativas que tienen los pacientes que reciben quimioterapia paliativa.

Método. Se realizaron búsquedas en PubMed desde el año 2000 hasta la actualidad. Artículos escritos en inglés y español. Un total de 20 artículos cumplieron los criterios de inclusión.

Resultados: Alrededor de la mitad de los pacientes que reciben quimioterapia paliativa, tienen creencias erróneas sobre las expectativas y objetivos del tratamiento. Los pacientes que entienden los objetivos del tratamiento presentan menor probabilidad de recibir quimioterapia al final de la vida, menores síntomas de ansiedad y depresión, mayor sensación de control y deseo de cuidados paliativos que se asocian a mejor calidad de vida.

Conclusión: un porcentaje significativo de pacientes oncológicos en fase avanzada, reciben quimioterapia paliativa sin conocer el objetivo del tratamiento con expectativas erróneas de curarse, lo que dificulta la participación en la toma de decisiones sobre su plan de cuidados.

Palabras clave: Quimioterapia paliativa; expectativas; cáncer avanzado; información; objetivos.

\section{[en] Information on palliative chemotherapy in patients with advanced cancer:} a review

Abstract. Introduction: Palliative chemotherapy is the first-line specific treatment in most oncology patients with advanced disease and it is essential for patients to understand the goals of treatment and expectations to fit the disease process.

Objective: To systematically review the beliefs and expectations of patients receiving palliative chemotherapy about treatment.

Method: We searched PubMed from 2000 to the present. Articles written in English and Spanish. A total of 20 articles met the inclusion criteria.

Results: About half of patients receiving palliative chemotherapy have misconceptions about treatment expectations and goals. Patients who understand the goals of treatment are less likely to receive chemotherapy at the end of life, less anxiety and depression, greater sense of control and desire for palliative care associated with better quality of life.

1 Unidad de Soporte Hospitalario de Cuidados Paliativos del Hospital Universitario de Getafe, Madrid.

E-mail: jnavarro7278@hotmail.es

2 Universidad Complutense de Madrid, Madrid.

3 Unidad de Soporte Hospitalario de Cuidados Paliativos del Hospital Universitario de Getafe, Madrid.

E-mail: mariajesus.boya@salud.madrid.org

* Dirección de correspondencia: José Miguel Navarro. Unidad de Soporte Hospitalario de Cuidados Paliativos del Hospital Universitario de Getafe. Ctra. Madrid - Toledo, Km 12,500, 28905 Getafe, Madrid.

E-mail: jnavarro7278@hotmail.es 
Conclusion: A significant percentage of advanced cancer patients receive palliative chemotherapy without knowing the purpose of treatment with erroneous expectations of cure, making it difficult to participate in decision making about their care plan.

Keywords: Palliative chemotherapy; expectations; advanced cancer; information; goals.

Sumario. 1. Introducción 2. Metodología 3. Resultados 3.1. Necesidades de Información 3.2. ¿Conocen los pacientes los objetivos de la quimioterapia paliativa? 3.3. Factores asociados al conocimiento del objetivo de la quimioterapia 3.4. Consecuencias de conocer el objetivo de la quimioterapia 4. Discusión 5. Conclusión 5.1. Limitaciones 6. Agradecimientos 7. Referencias bibliográficas

Cómo citar: Navarro Jiménez M, Cruzado JA, Boya Cristia MJ. Información sobre la quimioterapia paliativa en pacientes con cáncer avanzado: una revisión. Psicooncologia 2017; 14: 23-40. DOI: 10.5209/PSIC.55809

\section{Introducción}

El diagnóstico de cáncer es una de las noticias más devastadoras que se puede comunicar a un paciente y a su familia. Más aún en fases avanzadas, en las que incluso los avances de los tratamientos oncológicos en los últimos años, no posibilitan curar la enfermedad sino solo prolongar la supervivencia. Llega un momento en el que aparecen complicaciones inherentes a la propia enfermedad, buscar activamente un tratamiento específico, puede hacer más daño que bien, por lo que es planteable no tratarlas ${ }^{(1,2)}$. No obstante, hay tratamientos específicos como la quimioterapia paliativa, que pueden mejorar la calidad de vida del paciente, y está indicado hacerlo. Por lo tanto definir el objetivo del tratamiento es fundamental para los cuidados.

El cambio de tratamiento activo a quimioterapia paliativa es un proceso complejo, es difícil tanto para el profesional que tiene que comunicar el cese de tratamiento curativo, como para el paciente que en ocasiones toma conciencia de la gravedad de la enfermedad y la limitación temporal de la vida. Es necesario que los pacientes entiendan y acepten la naturaleza de la enfermedad, que conozcan los objetivos de los tratamientos paliativos y que sus expectativas de curación se ajusten a la realidad, para poder participar en la toma de decisiones y planificar la última etapa de su vida en función de sus deseos y preferencias. Los datos apuntan a que la mayoría de pacientes que reciben quimioterapia paliativa quieren ser informados sobre su tratamiento ${ }^{(3)}$, para poder participar en la toma de decisiones, generalmente de una manera compartida con su médico aunque existe variabilidad en las preferencias respecto al nivel de información y participación en la toma de decisiones ${ }^{(4-7)}$.

Una práctica clínica de calidad requiere que los pacientes formen parte de la toma de decisiones sobre sus cuidados, de acuerdo con sus preferencias y deseos. Para ello es fundamental que los pacientes oncológicos en fase avanzada conozcan su proceso de enfermedad y comprendan los objetivos de la quimioterapia paliativa. El propósito principal de este estudio es conocer la evidencia que existe sobre las creencias y expectativas que tienen los pacientes que reciben quimioterapia paliativa, describir las necesidades de información respecto al tratamiento y determinar los factores y las consecuencias de conocer el objetivo de la quimioterapia paliativa. 


\section{Metodología}

Se realizaron búsquedas en las bases de datos de PubMed, en abril y se actualizó en diciembre del 2016 utilizando diversos descriptores y términos en la estrategia de búsqueda. Para la revisión de artículos relacionados con el conocimiento que tienen los pacientes sobre los objetivos de la quimioterapia paliativa, seguimos esta estrategia:

Los límites que se aplicaron para la búsqueda, fueron: artículos publicados desde el año 2000 hasta diciembre del 2016. Escritos en inglés y/o castellano español. En humanos. Mayores de 19 años. No se aplicaron otros límites.

\#1 (chemotherapy[tiab] OR CANCER[tiab] OR NEOPLASMS[tiab]) AND (end of life[tiab] OR PALLIATIVE[tiab] OR ADVANCED[tiab] OR INCURABLE[tiab] OR TERMINAL[tiab] OR TERMINALLY[tiab] OR METASTATIC[tiab] OR METASTASIS[tiab]) AND (understanding[tiab] OR goal[tiab] OR goals[tiab] OR expectations[tiab] OR expectatives[tiab] OR curative[tiab] OR Cure[tiab]))

\#2 Neoplasms[mesh] AND ("Neoplasm Metastasis"[mesh] OR "neoplasms staging" [mesh] OR "Palliative Care"[mesh] OR "Terminal Care"[mesh] OR "Terminal ill"[mesh]) AND ("Health Knowledge, Attitudes, Practice"[mesh] OR "Truth Disclosure" OR Communication[mesh] OR "Decision making"[mesh]) \#3 \#1 OR \#2

\#4 (chemotherapy[tiab] OR CANCER[tiab] OR NEOPLASMS[tiab]) AND (end of life[tiab] OR PALLIATIVE[tiab] OR ADVANCED[tiab] OR INCURABLE[tiab] OR TERMINAL[tiab] OR TERMINALLY[tiab] OR METASTATIC[tiab] OR METASTASIS[tiab]) AND (understanding[tiab] OR goal[tiab] OR goals[tiab] OR expectations[tiab] OR expectatives[tiab] OR curative[tiab] OR Cure[tiab])) NOT medline[sb]

\#5 \#3 OR \#4

\section{Resultados}

En la búsqueda inicial se obtuvieron un total de 9.017 referencias en PubMed, revisamos el titulo y el abstract y muchas de ellas no eran relevantes para nuestro tema. Al final identificamos 116 artículos relevantes para nuestro objetivo. Descartamos 72 artículos que no cumplían los criterios de inclusión, (descritos más adelante), resultando un total de 44 artículos para análisis completo.

De estos excluimos 27 artículos: 9 artículos relacionados con la información; 8 sobre la participación en toma de decisiones; 9 a cerca de los cuidados, efectos y costes de la quimioterapia cerca de la muerte; y uno de opinión sobre el estudio de Week et al. ${ }^{(8)}$. Seleccionamos 17 trabajos que analizan forma específica las expectativas que tienen los pacientes sobre la quimioterapia paliativa y añadimos mediante búsqueda manual dos artículos mediante revisión de referencias, según la metodología de Cochrane Library.

En total, hemos extraído 19 artículos para nuestro trabajo. 
Los criterios de inclusión de los artículos fueron los siguientes:

- Artículos publicados desde principio del año 2000 hasta final del 2016. Escritos en castellano o inglés.

- Pacientes oncológicos con enfermedad avanzada (localmente avanzada o metastásica) $\geq 18$ años

- Artículos sobre el conocimiento de los objetivos de la quimioterapia paliativa.

- Tipos de estudios: todos, excepto en animales.

\subsection{Necesidades de Información}

Es frecuente que los pacientes oncológicos en fase avanzada de enfermedad deseen participar en la toma de decisiones sobre sus cuidados y para ello es necesario que estén informados. Necesitan tener una compresión exacta de su estado de enfermedad y sus opciones de tratamiento, por ello la mayoría de los pacientes, prefieren que sea el médico ${ }^{(8)}$ quien les explique el estado de su enfermedad ${ }^{(9)}$ de forma clara y honesta, incluso si las noticias son malas ${ }^{(10)}$.

En el estudio de Nowicki et al., se encontró que el $69 \%$ de los pacientes querían tener información ${ }^{(3)}$ sobre el tratamiento, y alrededor del $50 \%$ querían ser informados de las expectativas ${ }^{(3)}$, Según Oostendorp et al., el $74 \%$ de los pacientes desean información sobre la supervivencia ${ }^{(11)}$; Sin embargo, en el estudio de Elkin et al., son el $44 \%{ }^{(12)}$ de pacientes ancianos con cáncer colorectal metastásico, quienes desean recibir información sobre la supervivencia asociada al tratamiento de quimioterapia.

Jenkins et al. ${ }^{(13)}$ llevaron a cabo en el 2001 un trabajo sobre las necesidades de información, con una muestra de 2.331 pacientes. De ellos $940(40 \%)$ recibían tratamiento curativo y $841(36 \%)$ tratamiento paliativo y el resto estaban en remisión. Los datos mostraron que no hubo diferencias entre los grupos y el $97 \%$ de participantes querían recibir "toda" la información del tratamiento (efectos secundarios, cómo actúa y los objetivos del tratamiento etc.). Stewart et al., hallaron en un estudio con 105 mujeres con cáncer de ovario que el $90 \%$ querían información detallada sobre el tratamiento ${ }^{(14)}$, lo que coincide con Detmar et al., que informan que más del $95 \%$ de pacientes demandan recibir información sobre la quimioterapia paliativa que reciben ${ }^{(9)}$.

Comprobamos que la mayoría de los pacientes oncológicos en fase avanzada, desean tener información en mayor o menor medida ${ }^{(5)}$ sobre el tratamiento y son los médicos los responsables de cubrir estas necesidades de información. Según la bibliografía, Gatellari et al., valoraron la información que recibían los pacientes y encontraron que el $84,7 \%$ recibían información sobre el objetivo del tratamiento, en un $73,7 \%$ de los inconvenientes y efectos secundarios, que era una enfermedad incurable en el $74,6 \%$, de las expectativas de vida en el 57,6\% y el 44,1\% de los casos sobre los tratamientos alternativos ${ }^{(15)}$. Resultados similares al estudio de Kooedot, et al. que afirman que el $75 \%$ de pacientes fueron informados sobre el objetivo del tratamiento, el 58\% sobre su esperanza de vida y solo el $33 \%$ fueron informados sobre opciones alternativas del tratamiento, hay datos que confirma que los oncólogos pasan más tiempo hablando sobre tratamientos activos contra el cáncer, que tratamientos de soporte ${ }^{(16)}$. También es frecuente la escasez de información que ofrecen los oncólogos sobre la supervivencia de la quimioterapia paliativa, en la mayoría de consultas se habla de forma vaga o inexistente, Suzanne et al., encontraron que solo a 3 de los 23 pacientes se les mostraban datos numéricos en relación a la supervivencia ${ }^{(17)}$. 
Aún siendo en ocasiones insuficiente la información dada, los resultados dicen que la mayoría de pacientes expresaron un alto nivel de satisfacción con la información recibida $^{(18)}$, reconocieron estar bien informados ${ }^{(19)}$ y encontraron que la información era útil para ellos ${ }^{(10)}$. Según los pacientes el proporcionarles más información no aumentó los niveles de ansiedad, no disminuyó la esperanza, ni elevó el malestar emocional $^{(20)}$.

En resumen, la mayoría de los pacientes desean recibir información, siendo el objetivo del tratamiento el tema más demandado y con mayor respuesta por parte de los oncólogos. Diferente a la información sobre la supervivencia y tratamientos no curativos que son los que menor cobertura reciben.

\section{2. ¿Conocen los pacientes los objetivos de la quimioterapia paliativa?}

En la tabla 1 se exponen los resultados y características de los 19 estudios seleccionados a través de la revisión bibliográfica realizada para el presente estudio.

Hemos identificado en la literatura un total de 19 trabajos que exploraron el conocimiento que tenían los pacientes oncológicos en fase avanzada sobre los objetivos y las expectativas de la quimioterapia paliativa, con mayor incidencia en EEUU, Australia, Italia y en los últimos años en Corea. La mayoría de los resultados muestran que alrededor de la mitad ${ }^{(10,22-23)}$ de los pacientes que recibían quimioterapia paliativa, tenían creencias erróneas sobre los objetivos del tratamiento, llegando hasta el $80 \%$ en uno de los estudios ${ }^{(24)}$.

En un trabajo realizado en Italia el 8\% de pacientes en cuidados paliativos, creían que su tratamiento era para problemas distintos al cáncer ("problemas de espalda" "pólipos" "problemas de páncreas")(21). Hay datos que afirman que alrededor del $50 \%$ de pacientes aseguraban que el objetivo del tratamiento era la curación ${ }^{(15,21,25,26)}$, y en el $70 \%$ de los casos las expectativas eran que "desaparezca todo el cáncer" (27).

Con el objetivo de determinar si los pacientes en fase avanzada entendían correctamente que el tratamiento no era curativo, Craft et al., en 2005, llevaron a cabo un estudio longitudinal ${ }^{(22)}$, los resultados en la línea base indicaban que el $30,4 \%$ de los pacientes creían que el objetivo era curarse, y $19,1 \%$ de los pacientes que recibieron quimioterapia paliativa desconocían el objetivo del tratamiento, a las 12 semanas el 52,1\% tenían creencias erróneas o desconocían el objetivo de la quimioterapia ${ }^{(22)}$. Posteriormente en el año 2007, Burns et al., ${ }^{(28)}$ compararon las creencias entre el paciente y su cuidador y solo el $37 \%$ coincidían en que el tratamiento no era curativo.

En la misma línea, Week et al. realizaron en 2012 un estudio multicéntrico con 1.193 pacientes oncológicos con enfermedad avanzada, encontraron que el $69 \%$ de pacientes con cáncer de pulmón y el $81 \%$ de pacientes con cáncer colorectal no entendían que la quimioterapia no fuera a curar su cáncer, siendo mayor el error en cáncer colorectal ${ }^{(8,29)}$. También en EEUU en 2015, Marck et al. evaluaron las expectativas del objetivo del tratamiento y concluyeron que el $33 \%$ de pacientes reconocían que era "nada probable" curar su cáncer, de ellos el $18 \%$ recibieron quimioterapia el último mes de vida y expresaban que "no era en absoluto probable que curara su cáncer" (30). 


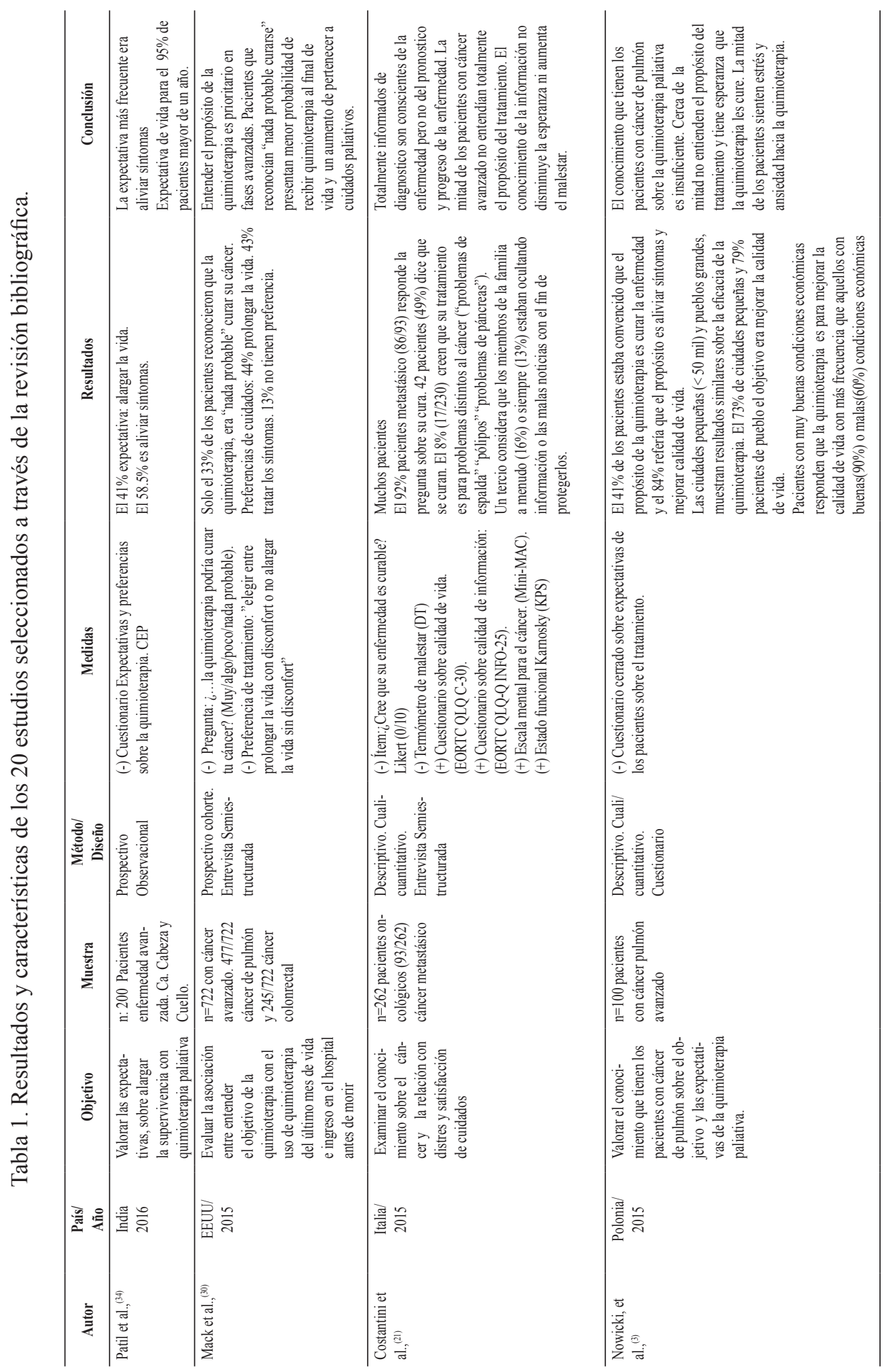




\begin{tabular}{|c|c|c|c|}
\hline 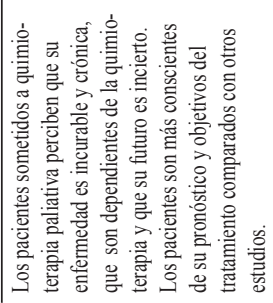 & 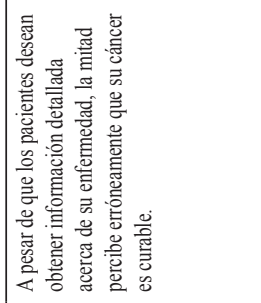 & 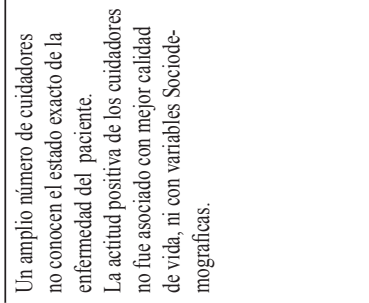 & 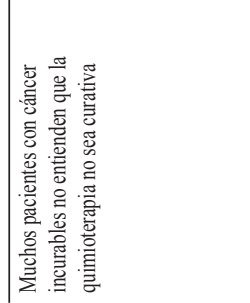 \\
\hline 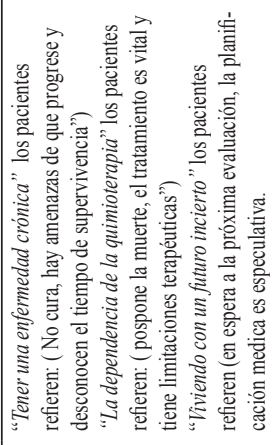 & 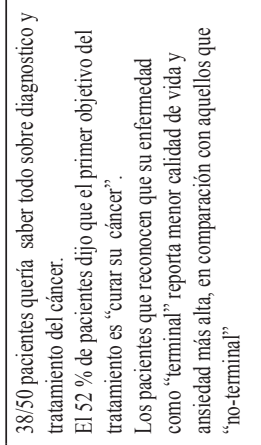 & 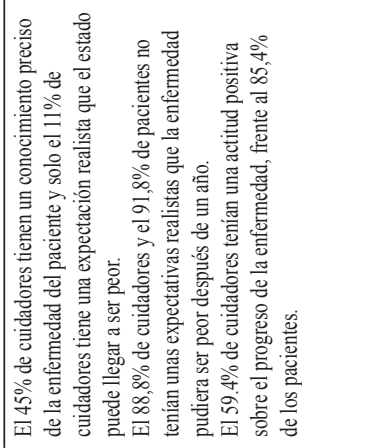 & 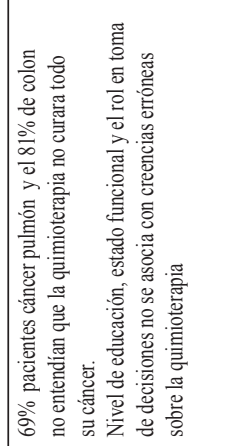 \\
\hline 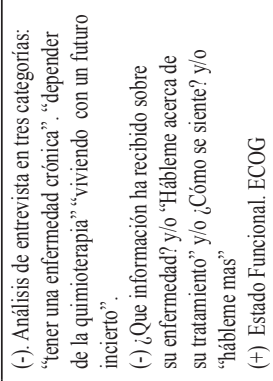 & 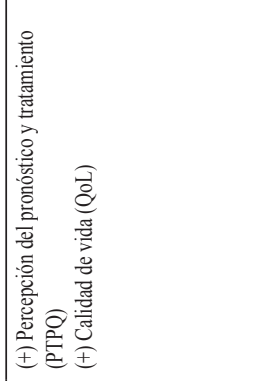 & 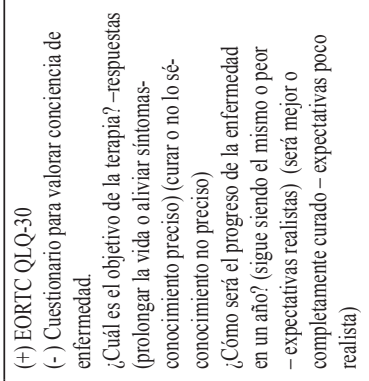 & \\
\hline 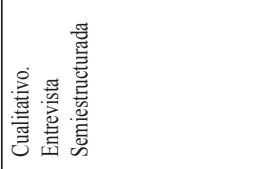 & 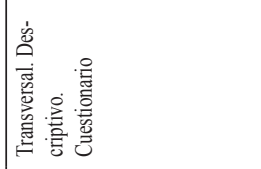 & 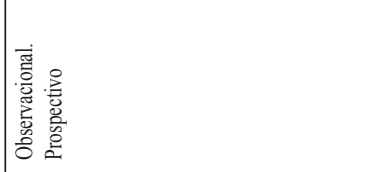 & 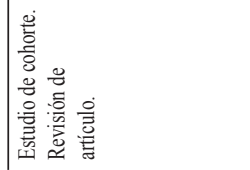 \\
\hline 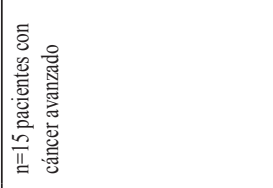 & 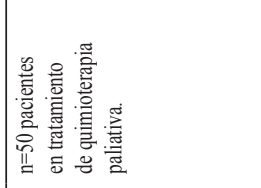 & 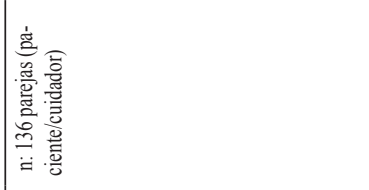 & 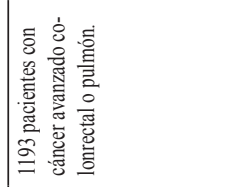 \\
\hline 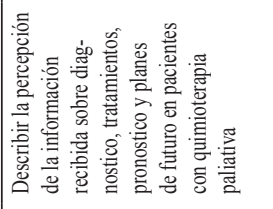 & 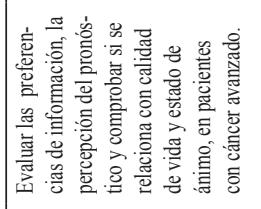 & 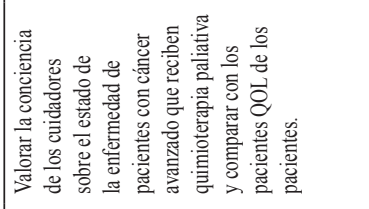 & 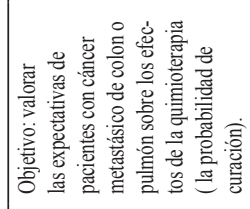 \\
\hline 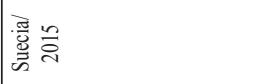 & 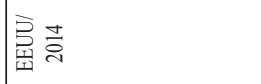 & 总 壳 & 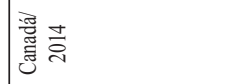 \\
\hline 害手。 & 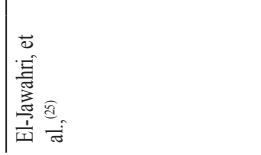 & 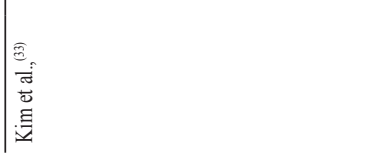 & 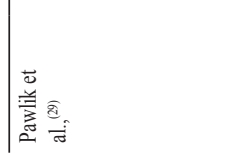 \\
\hline
\end{tabular}




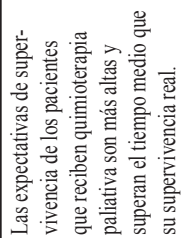

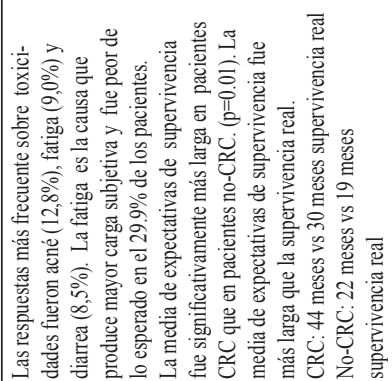

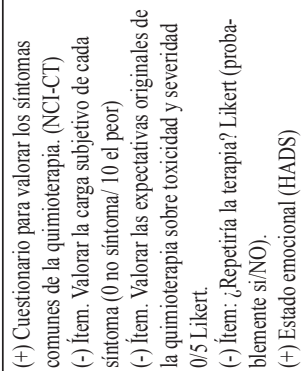

摇

产高

总富

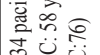

恶苞

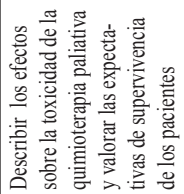

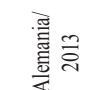

离

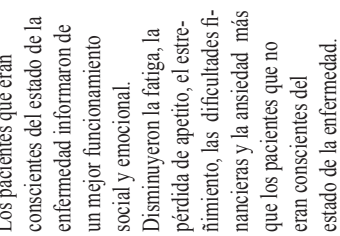

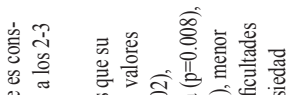

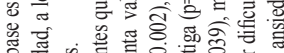

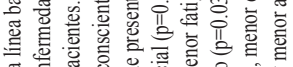

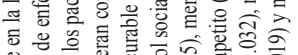

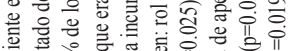

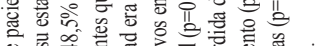

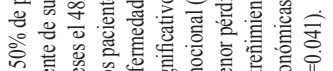

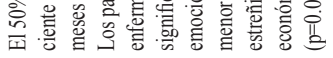

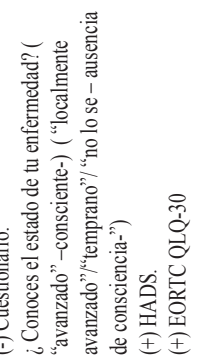

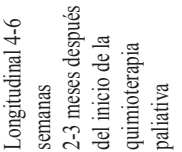

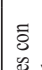

这 률

产.

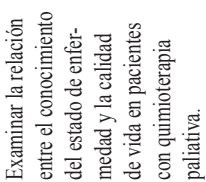

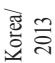

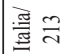

离

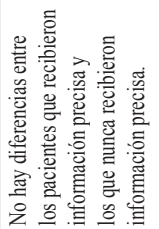

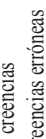

형

s

这 를

하응

$\therefore$ 要

差总

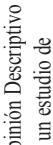

亭言 흥

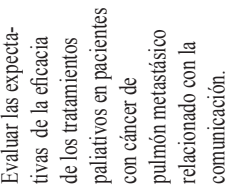

昰

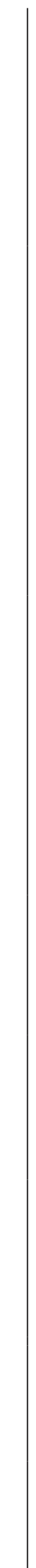




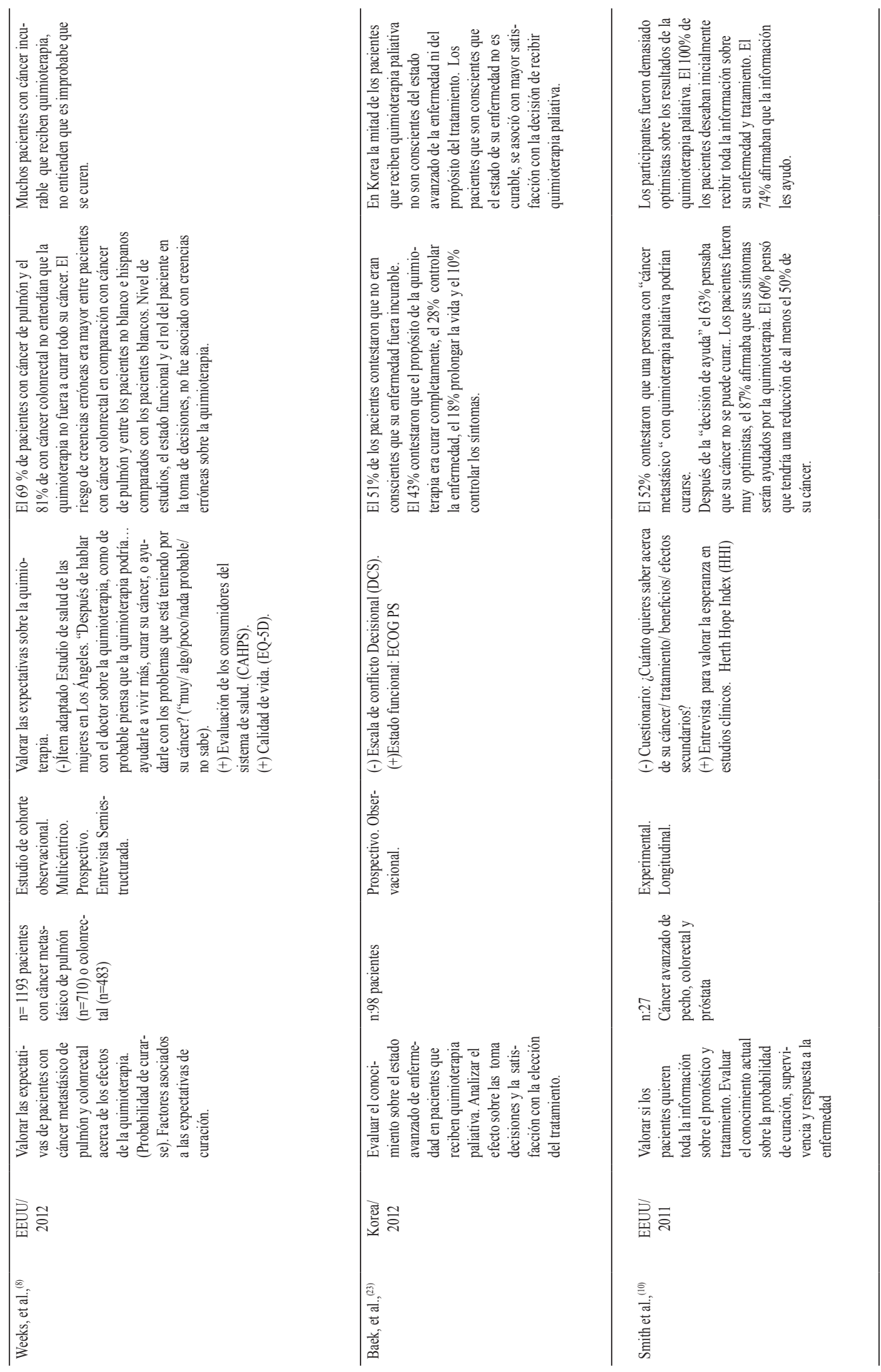




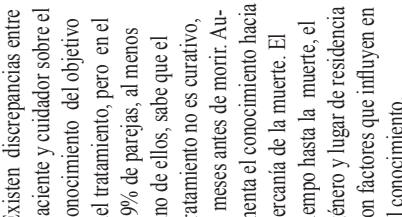
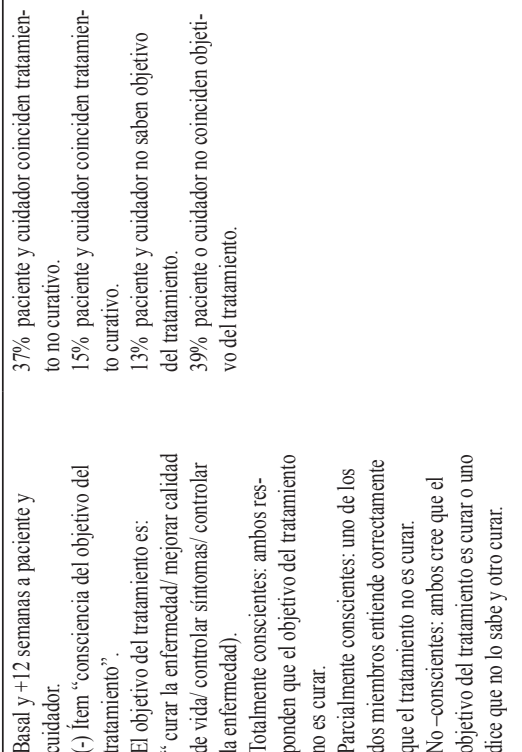

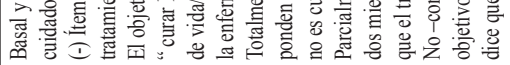

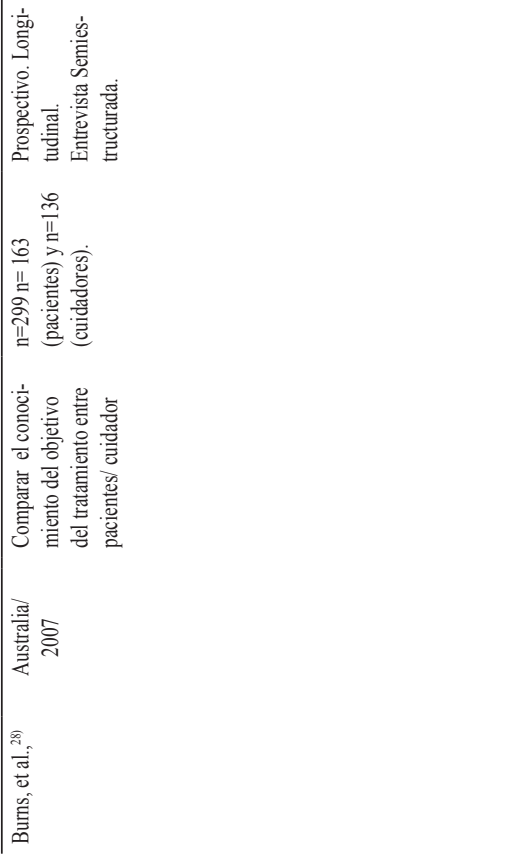

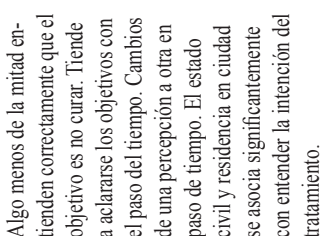
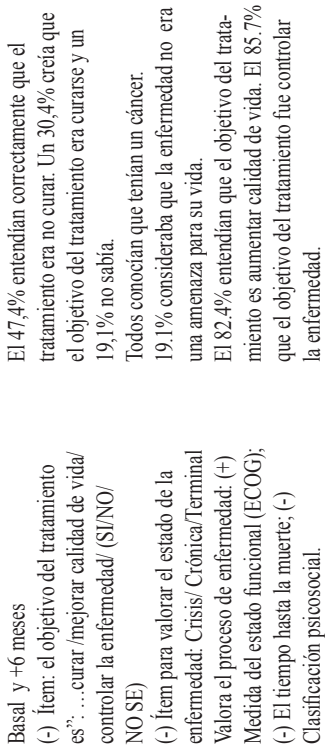

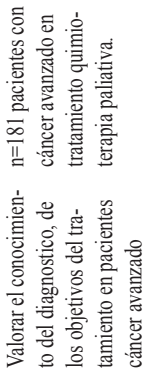

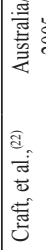




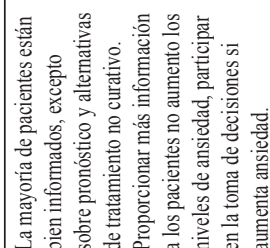

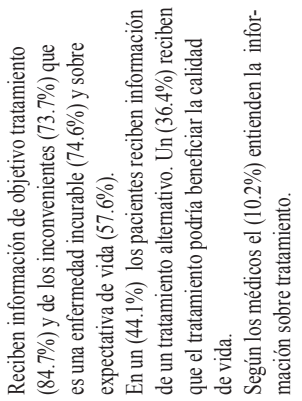

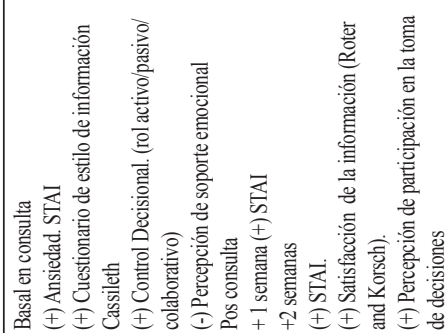

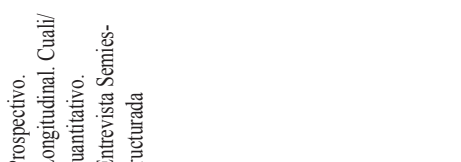

总总器畐总

言

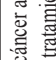

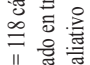

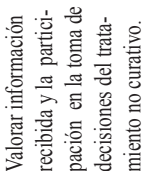

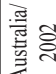

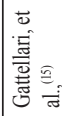

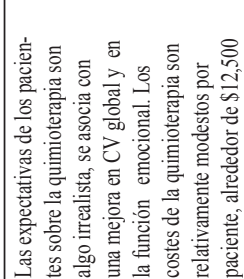

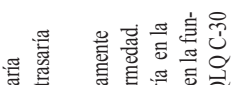

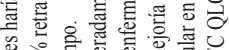

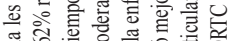

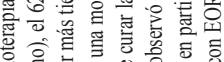

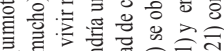

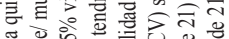

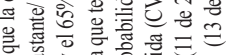

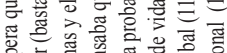

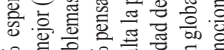

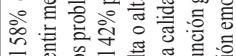

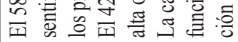

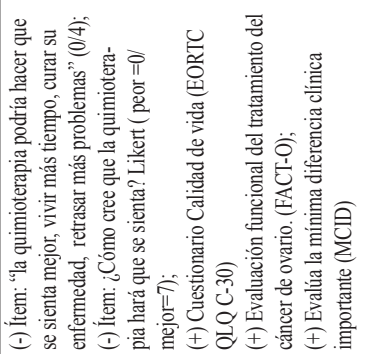

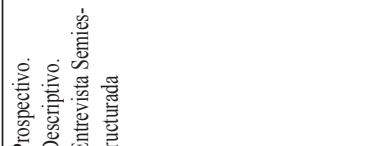

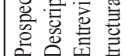

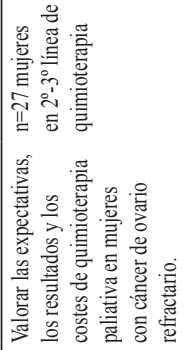

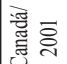

这

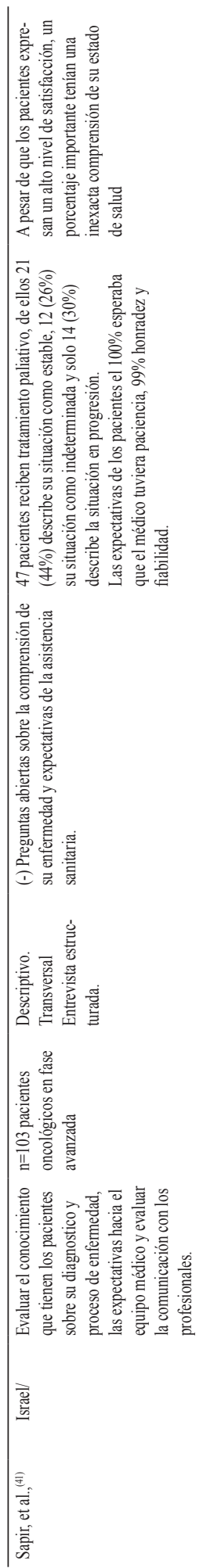


En el año 2015, Martisson et al. ${ }^{(31)}$ desarrollaron un estudio cualitativo que mediante una entrevista semiestructurada clasificaba en tres categorías la percepción que tenían los pacientes de la enfermedad: "tener una enfermedad crónica" y/o "la dependencia de la quimioterapia" y/o "viviendo con un futuro incierto". Los resultados comparados con otros estudios muestran que los pacientes que eran más conscientes de su pronóstico y objetivos del tratamiento, percibían su enfermedad como incurable, crónica, con un futuro incierto y dependientes de la quimioterapia.

Destaca el creciente interés sobre el tema en países asiáticos en los últimos años, encontramos tres trabajos en $\mathrm{Corea}^{(23,32-33)}$, y el más actual, publicado en 2016 en la India. Los resultados coinciden con los publicados en países de occidente y EEUU, alrededor de la mitad de los pacientes tienen creencias erróneas sobre los objetivos de la quimioterapia paliativa ${ }^{(23)}$. Según un estudio coreano, el $43 \%$ de los pacientes contestaron que el propósito de la quimioterapia era curar completamente, el $28 \%$ controlar la enfermedad, el $18 \%$ prolongar la vida y el $10 \%$ controlar la enfermedad ${ }^{(23)}$.

El reciente trabajo de Patil et al., ${ }^{(34)}$ realizado en la India, con pacientes oncológicos de cabeza y cuello muestra que el $41 \%$ de los pacientes esperaba que la quimioterapia les prolongaría la vida, para el 58,5\% sus expectativas eran aliviar síntomas y el incremento mínimo de expectativa de vida al recibir la quimioterapia era mayor de un año para el $94,5 \%$ de los pacientes.

En conclusión, los datos confirman, que entre el $40-80 \%$ de los pacientes que reciben quimioterapia paliativa tienen creencias erróneas sobre los objetivos y expectativas del tratamiento.

\subsection{Factores asociados al conocimiento del objetivo de la quimioterapia}

Encontramos estudios que examinaban la relación entre diferentes factores y el conocimiento de los objetivos de la quimioterapia paliativa. Según los datos, las diferencias étnicas y culturales se asocian con entender el objetivo del tratamiento, el riesgo de creencias erróneas era mayor entre los pacientes de raza no blanca e hispanos en comparación con los caucásicos ${ }^{(8)}$. Respecto al lugar de residencia, los resultados fueron que los sujetos que residían en áreas rurales era más probable que desconocieran el objetivo de su tratamiento ${ }^{(22)}$. Sin embargo, en el estudio de Nowicki et al., ${ }^{(3)}$ no se encontraron diferencias entre los habitantes de las ciudades pequeñas y grandes, el $73 \%$ de los habitantes de las ciudades pequeñas y el 79\% de los habitantes de las grandes pensaron que la quimioterapia era para mejorar la calidad de vida.

Otros estudios observaron las variables clínicas y los resultados fueron, que tener un buen pronostico ${ }^{(15)}$ se relacionaba con un mejor conocimiento de los objetivos de tratamiento y que los pacientes con cáncer colonrectal tenían mayor riesgo de informar creencias inexactas sobre la quimioterapia paliativa en comparación con pacientes con cáncer de pulmón. Week et al., ${ }^{(8)}$ analizaron la relación con el estado funcional y los resultados no fueron concluyentes, al igual que el rol del paciente en la toma de decisiones, que tampoco parece influir ${ }^{(29)}$.

Otro factor importante, es el tiempo hasta la muerte. Las investigaciones coinciden en señalar que se relaciona con la compresión de los objetivos del tratamiento. Burns et al., en un estudio de pares paciente-cuidador, encontraron que 6 meses antes de la muerte el $89 \%$ de los casos al menos uno de ellos comprendía que el tratamiento no 
era curativo ${ }^{(28)}$. Asimismo, en el estudio de Craft et al., informan que el $66 \%$ de los pacientes, con esperanza de vida menor de 6 meses comprendían que la quimioterapia paliativa no tenía propósito curativo ${ }^{(22)}$, lo que muestra que con el paso del tiempo se tiende a aclarar los objetivos del tratamiento.

Los datos apuntan a que factores como la cercanía a la muerte ${ }^{(22,28)}$, el residir en ciudades y ser de etnia blanca ${ }^{(8)}$, se relacionan con un mejor conocimiento de los objetivos de la quimioterapia paliativa.

\subsection{Consecuencias de conocer el objetivo de la quimioterapia}

La quimioterapia paliativa es la primera elección de tratamiento oncoespecífico en la mayoría de pacientes con cáncer avanzado. Con frecuencia los pacientes con cáncer incurable se someten a tratamientos sin entender totalmente la información de su situación ${ }^{(8)}$. Están dispuestos a recibir quimioterapia aún con altos niveles de toxicidad a pesar de obtener pequeños beneficios ${ }^{(35,36)}$. Así, el número de pacientes que reciben quimioterapia cerca de la muerte está incrementando ${ }^{(36)}$, entre el 20 y el $50 \%$ de pacientes reciben la quimioterapia dentro de los 30 días antes de la muerte y hasta un $56 \%$ en los últimos seis meses $^{(25)}$. Por lo tanto, es prioritario entender el propósito del tratamiento porque los pacientes que reconocían: "nada probable curarse", presentaban menor probabilidad de recibir quimioterapia al final de vida ${ }^{(27,30,37)}$.

Los pacientes que prolongan el tratamiento de quimioterapia es menos probable que conozcan que su enfermedad es terminal y que expresen sus deseos sobre los cuidados a los médicos. Disminuyendo la probabilidad de morir en casa o en su lugar preferido e incrementan la posibilidad de morir en una unidad de cuidados intensivos y aceptar terapias más agresivas ${ }^{(27,37)}$ que se asocian como un indicador de mala calidad de cuidados ${ }^{(38)}$ y una "mala muerte" ${ }^{(10)}$.

Los resultados indican que los pacientes con creencias erróneas sobre los objetivos del tratamiento tendían a sobreestimar su pronóstico ${ }^{(8,27)}$ y su prolongación de $\operatorname{vida}^{(15,38)}$, siendo las expectativas irreales y superando el tiempo medio de supervivencia real ${ }^{(40)}$. El desconocimiento del proceso de enfermedad se ha asociado a mayor nivel de estrés ${ }^{(7,39)}$.

Sin embargo, los pacientes que reconocen que la quimioterapia no les cura, y comprenden la naturaleza incurable del cáncer que padecen, desean recibir cuidados paliativos $^{(30,37)}$, estos se asocian con asistencia de calidad en los cuidados cerca de la muerte y con mejor bienestar. Los pacientes que eran más conscientes de la gravedad y estado de su enfermedad presentaban mayor sensación de control, más facilidad para planificar ${ }^{(30,40)}$, mejor funcionamiento social y emocional ${ }^{(32)} \mathrm{y}$ disminuyeron la fatiga, la pérdida de apetito, el estreñimiento y las dificultades económicas, también reconocían, menor ansiedad y depresión ${ }^{(30,32,40)}$.

También es importante para el profesional que el paciente comprenda el proceso de enfermedad y las metas del tratamiento, porque ayuda a transmitir las decisiones clínicas a los pacientes con esperanza de vida corta ${ }^{(27)}$.

En conclusión, la mayoría de estudios apuntan que entender los objetivos del tratamiento y tener las expectativas reales es beneficioso tanto para el paciente, como para los profesionales, en fase avanzada de enfermedad ${ }^{(8,27,38)}$. 


\section{Discusión}

En la literatura desde el año 2000 hasta la actualidad, hemos identificado un total de 19 publicaciones que valoran el conocimiento de los objetivos que tienen los pacientes oncológicos en fase avanzada sobre la quimioterapia paliativa. Se trata de una revisión sistemática, con un estudio experimental ${ }^{(10)}$ y el resto son estudios descriptivos - cualitativos. Para la recogida de datos se emplearon instrumentos no validados, los datos se recopilaron mediante cuestionarios y/o entrevistas semiestructuradas, creadas para cada trabajo de forma independiente.

Los trabajos se han llevado a cabo distintas décadas y en diferentes países, siendo los más frecuentes en EEUU (5), Australia (3), Italia (2), Canadá (2) y los más actuales en $\mathrm{Corea}^{(3)}$. Es importante destacar, que aún tratándose de trabajos desarrollados en distintas décadas ${ }^{(41)} \mathrm{y}$ en diferentes países y culturas los datos son muy similares, alrededor de la mitad de los pacientes que reciben quimioterapia no conocen el objetivo del tratamiento y sus expectativas de curación son erróneas.

En todos los estudios, son pacientes oncológicos en fase avanzada de enfermedad que reciben quimioterapia paliativa, son muestras pequeñas excepto en el trabajo de Week et al., ${ }^{(8)}$ que recogen la información en 1.193 pacientes y su revisión ${ }^{(29)}$ La población elegida presenta diferentes tipos de cáncer, siendo los estudios de pacientes con cáncer de pulmón (3), y pacientes con cáncer de pulmón o colorectal los más frecuentes (3). El resto de trabajos son población con cáncer de cabeza y cuello, ovario, gastrointestinal, pecho y próstata y en nueve no se determina el tipo de cáncer. Los datos más concluyentes los encontramos en pacientes con cáncer de pulmón, donde un alto porcentaje de pacientes, presentan unas creencias erróneas sobre el tratamiento y afirmaban que la quimioterapia paliativa curaba su cáncer ${ }^{(5,8,27)}$.

En este punto de la enfermedad, cada vez es mayor el número de pacientes que deciden recibir quimioterapia cerca de la muerte ${ }^{(36)}$, aún con pequeños beneficios $\mathrm{y}$ alta toxicidad ${ }^{(35,36)}$. Es frecuente que los pacientes decidan recibir tratamiento agresivo, por no entender los objetivos del tratamiento o porque sus expectativas de curación son erróneas. Entender el propósito del tratamiento es prioritario en fase avanzada de enfermedad para participar en la toma de decisiones, factores como la cercanía a la muerte ${ }^{(22,28)}$ el residir en ciudades y ser de etnia blanca ${ }^{(8)}$, se relacionan con un mejor conocimiento. Los pacientes que presentaban un buen entendimiento tenían mayor sensación de control, más facilidad para planificar, menor ansiedad-depresión y mejor calidad de vida cerca de la muerte ${ }^{(18,19,40)}$, mientras que el desconocimiento se asoció a mayor estrés ${ }^{(7,18)}$ y a unas expectativas irreales que sobreestiman el tiempo de supervivencia, dificultando la planificación del los cuidados.

En resumen, el interés sobre el conocimiento de las expectativas que tienen los pacientes sobre quimioterapia paliativa ha sido un tema latente durante décadas. Destaca que a pesar del paso del tiempo y la evolución en la relación médico paciente, no hayamos encontrado cambios significativos sobre el conocimiento de los objetivos del tratamiento. También resalta que aun existiendo diferencias sanitarias y culturales entre los países, los datos coinciden que alrededor de la mitad de los pacientes desconocen el objetivo de la quimioterapia paliativa.

No obstante, aun siendo datos concluyentes hay que tratarlos con cautela, porque en cada estudio se creó un instrumento de medida de forma independiente. 
Por ello uno de nuestros intereses para la práctica clinica, es la creación de una herramienta, que permita recoger las expectativas que tienen los pacientes de forma metódica y homogénea.

\section{Conclusión}

En conclusión en fase avanzada de enfermedad la quimioterapia paliativa es el tratamiento de primera elección, y alrededor de la mitad de los pacientes reciben tratamiento sin entender los objetivos del tratamiento ${ }^{(15,21,25,26,41)}$. Son muchas las posibles razones y ninguna concreta de tales malentendido, desde que los oncólogos no den la información por falta de tiempo ${ }^{(42)}$, por proteger al paciente, por mantener la esperanza de los pacientes ${ }^{(43)}$ etc., hasta que los pacientes no entiendan la información, la olviden o la rechacen como parte de un proceso de negación ${ }^{(15)}$.

La evidencia muestra que para una asistencia de calidad, donde los pacientes puedan participar en la toma de decisiones sobre sus cuidados, como es el deseo de la mayoría de los pacientes, participar de forma compartida ${ }^{(44)}$. Resulta prioritario que entiendan el objetivo del tratamiento y la naturaleza de la enfermedad porque el entendimiento erróneo puede comprometer la validez de las decisiones. Por ello nuestro interés en seguir investigando sobre este tema, con el objetivo de conocer las expectativas reales con la intención de reducir o eliminar el número de pacientes que deciden recibir quimioterapia paliativa sin conocer el objetivo del tratamiento.

\subsection{Limitaciones}

No se trata de un estudio meta-analítico, con datos cuantitativos. La mayoría son estudios descriptivos-cualitativos. Para la recogida de datos se emplearon instrumentos no validados, los datos se recopilaron mediante cuestionarios y/o entrevistas semiestructuradas, creadas para cada trabajo de forma independiente.

La búsqueda automatizada nunca encuentra todos los artículos elegibles.

\section{Agradecimientos}

A $D^{a}$ Concepción Campos Asensio por su gran ayuda y colaboración en cada momento de consulta. Al ESH de Cuidados Paliativos del Hospital de Getafe por poder contar siempre con su ayuda y dedicación. A $D^{o}$ Iván Jiménez Vera ${ }^{\dagger}$ por enseñarnos e inspirarnos en la idea.

\section{Referencias bibliográficas}

1. Grassi L. Communicating anticancer treatment cessation and transition to palliative care: The need for a comprehensive and culturally relevant, person-centered approach. Cancer 2015;121:4104-7. Doi: 10.1002/cncr.29638.

2. Alonso A, Mora M. El tratamiento de las infecciones al final de la vida. ¿Puede estar indicada la adecuación del esfuerzo terapéutico? Treating end-of-life infections. Is optimizing life-sustaining treatment an option? Revista eidon.es. 2015. 
3. Nowicki A, Woźniak K, Krajnik M. Understanding the purpose of treatment and expectations in patients with inoperable lung cancer treated with palliative chemotherapy. Contemp Oncol (Pozn) 2015; 19:333-7. Doi: 10.5114/wo.2015.53249

4. Evans N, Pasman HRW, Payne SA, Seymou J, Pleschberger S, Deschepper R, et al. Older patients' attitudes towards and experiences of patient-physician end-of-life communication: A secondary analysis of interviews from British, Dutch and Belgian patients. BMC Paliat Care 2012;11:1. Doi: 10.1186/1472-684X-11-24.

5. Brom L, Hopmans W, Pasman HR, Timmermans DR, Widdershoven GA, OnwuteakaPhilipsen BD. Congruence between patients' preferred and perceived participation in medical decision-making: A review of the literature. BMC Med Inform Decis Mak 2014. Doi: 10.1186/1472-6947-14-25.

6. Nuñez Olarte JM. Información al paciente en situación terminal: diferencias entre las publicaciones anglosajonas y la experiencia diaria en España. Med Paliat 2014;21:11320. Doi:10.1016/j.medipa.2012.11.004

7. Innes $\mathrm{S}$, Payne S. Advanced cancer patients' prognostic information preferences: a review J Palliat Med 2009;23:29-39. Doi: 10.1177/0269216308098799.

8. Weeks JC, Catalano PJ, Cronin A, Finkelman MD, Mack JW, Keating NL, et al. Patients' expectations about effects of chemotherapy for advanced cancer. N Engl J Med 2012;367: 1616-25. Doi: 10.1056/NEJMoa1204410

9. Detmar SB, Aaronson NK, Wever LDV, Muller M, Schornagel JH. How are you feeling? Who wants to know? Patients' and oncologists' preferences for discussing health-related quality-of-life issues. J Clin Oncol 2000;18:3295-301. Doi:10.1200/jco.2000.18.18.3295

10. Smith TJ, Dow LA, Virago EA, Khatcheressian J, Matsuyama R, Lyckholm LJ. A pilot trial of decision aids to give truthful prognostic and treatment information to chemotherapy patients with advanced cancer. J Clin Oncol 2011;9: 79.

11. Oostendorp LJ, Ottevanger PB, Wouw AJ, Schoenaker IJ, Graaf H, Graaf WT, et al. Expected survival with and without second-line palliative chemotherapy: who wants to know? Health Expectations 2015;18:2903-14. Doi: 10.1111/hex.12275

12. Elkin EB, Kim SH, Casper ES, Kissane DW, Schrag D. Desire for information and involvement in treatment decisions: elderly cancer patients' preferences and their physicians perceptions. J Clin Oncol 2007;25:5275-80. Doi:10.1200/JCO.2007.11.1922

13. Jenkins V, Fallowfield L, Saul J. Information needs of patients with cancer: results from a large study in UK cancer centers. Br J Cancer 2001;84:48-5 Doi:10.1054/bjoc.2000.1573

14. Stewart DE, Wong F, Cheung AM, Dancey J, Meana M, Cameron JI, Rosen B. Information needs and decisional preferences among women with ovarian cancer. Gynecol Oncol 2000;77: 357-61. Doi:10.1006/gyno.2000.5799

15. Gattellari M, Voigt KJ, Butow PN, Tattersall MH. When the treatment goal is not cure: Are cancer patients equipped to make informed decisions? J Clin Oncol 2002;20:503-13. Doi:10.1200/JCO.2002.20.2.503

16. Koedoot CG, Oort,FJ, De Haan RJ, Bakker PJM, De Graeff A, De Haes JCJM. The content and amount of information given by medical oncologists when telling patients with advanced cancer what their treatment options are: palliative chemotherapy and watchful-waiting. Eur J Cancer 2004;40:225-35. Doi: 10.1016/j.ejca.2003.10.008

17. Audrey S, Abel J, Blazeby JM, Falk S, Campbell R. What oncologists tell patients about survival benefits of palliative chemotherapy and implications for informed consent: qualitative study. BMJ 2008;337:a752. Doi:10.1136/bmj.a752

18. Buckman R. Communications and emotions. BMJ 2002;325:672-72. Doi: 10.1136/ bmj.325.7366.672 
19. Tattersall MH, Gattellari M, Voigt K, Butow PN. Retraction note: When the treatment goal is not cure: Are patients informed adequately? Support Care Cancer 2002;10:314-21. Doi 10.1007/s005200100291

20. Eidinger RN, Schapira DV. Cancer patients' insight into their treatment, prognosis, and unconventional therapies. Cancer 1984;53:2736-40. Doi:10.1002/10970142(19840615)53:12<2736::AID-CNCR2820531233>3.0.CO;2-W

21. Costantini, A, Grassi L, Picardi A, Brunetti S, Caruso R, Nanni MG, et al. Awareness of cancer, satisfaction with care, emotional distress, and adjustment to illness: an Italian multicenter study. Psychooncology 2015; 24:1088-96. Doi: 10.1002/pon.3768

22. Craft PS, Burns CM, Smith WT, Broom DH. Knowledge of treatment intent among patients with advanced cancer: A longitudinal study. Eur J Cancer Care 2005;14:417-25. Doi10.1111/j.1365-2354.2005.00601.X

23. Baek SK, Kim SY, Heo DS, Yun YH, Lee MK. Effect of advanced cancer patients' awareness of disease status on treatment decisional conflicts and satisfaction during palliative chemotherapy: A Korean prospective cohort study. Support Care Cancer 2012;20:130916. Doi: $10.1007 / \mathrm{s} 00520-011-1218-5$

24. Passaro A, de Marinis F. Expectations as outcome of effectiveness for patients with metastatic lung cancer. Ann Palliat Med 2013;2:164-6. Doi: 10.3978/j.issn.22245820.2013.09.01

25. El-Jawahri A, Traeger L, Park ER, Greer JA, Pirl WF, Lennes IT, et al. Associations among prognostic understanding, quality of life, and mood in patients with advanced cancer. Cancer 2014:120:278-85. Doi: 10.1002/cncr.28369.

26. Doyle C, Crump M, Pintilie M Oza AM. Does palliative chemotherapy palliative? Evaluation of expectations, outcomes, and costs in women receiving chemotherapy for advanced ovarian cancer. J Clin Oncol 2001;19: 1266-74. Doi:10.1200/ JCO.2001.19.5.1266

27. Temel JS, Greer JA, Admane S, Gallagher ER, Jackson VA, Lynch TJ, et al. Longitudinal perceptions of prognosis and goals of therapy in patients with metastatic non-smallcell lung cancer: Results of a randomized study of early palliative care. J Clin Oncol 2011;29:2319-26. Doi: 10.1200/JCO.2010.32.4459

28. Burns CM, Broom DH, Smith WT, Dear K, Craft PS. Fluctuating awareness of treatment goals among patients and their caregivers: A longitudinal study of a dynamic process. Support Care Cancer 2007;15:187-96. Doi: 10.1007/s00520-006-0116-8

29. Pawlik TM, Devon KM, Fields CA, Hinshaw DB. Evidence-based reviews in surgery group. What are patients' expectations about the effects of chemotherapy for advanced cancer? J Amer College of Surgeons 2014; 219:588-90. Doi:10.1016/j.jamcollsurg.2014.06.008

30. Mack JW, Walling A, Dy S, Antonio ALM, Adams J, Keating NL, et al. Patient beliefs that chemotherapy may be curative and care received at the end of life among patients with metastatic lung and colorectal cancer. Cancer 2015; 121:1891-7. Doi:10.1002/cncr.29250

31. Martinsson L, Axelsson B, Melin-Johansson C. Patients' perspectives on information from physicians during palliative chemotherapy: A qualitative study. Palliat Support Care 2015; 14:1-8. Doi:10.1017/S1478951515001200

32. Lee MK, Baek SK, Kim SY, Heo DS, Yun YH, Park SR, et al Awareness of incurable cancer status and health-related quality of life among advanced cancer patients: a prospective cohort study. J Palliat Med 2013;27:144-54. Doi:10.1177/0269216311429042

33. Kim S, Ko Y, Kwon S, Shin DY, Kim CH, Yang SH, Na I. Family caregivers' awareness of illness and attitude toward disclosure during chemotherapy for advanced cancer. Psychooncology, 2014;23:1300-1306. Doi:10.1002/pon.3565 
34. Patil V, Joshi A, Noronha V, Deodhar J, Bhattacharjee A, Dhumal S, et al. Expectations and preferences for palliative chemotherapy in head and neck cancers patients. Oral Oncol 2016;63:10-5. Doi:10.1016/j.oraloncology.2016.10.023

35. Slevin ML, Stubbs L, Plant HJ, Wilson P, Gregory WM, Armes PJ, et al. Attitudes to chemotherapy: Comparing views of patients with cancer with those of doctors, nurses, and general public. BMJ 1990;300:1458-60

36. Matsuyama R, Reddy S, Smith TJ. Why do patients choose chemotherapy near the end of life? A review of the perspective of those facing death from cancer. J Clin Oncol 2006;24:3490-96. Doi:10.1200/JCO.2005.03.6236

37. Wright AA, Zhang B, Ray A, Mack JW, Trice E, Balboni T, et al. Associations between end-of-life discussions, patient mental health, medical care near death, and caregiver bereavement adjustment. JAMA 2008;300: 1665-73. Doi: 10.1001/jama.300.14.1665.

38. Earle CC, Landrum MB, Souza JM, Neville BA, Weeks JC, Ayanian JZ. Aggressiveness of cancer care near the end of life: Is it a quality-of-care issue? J Clin Oncol 2008;26:38606. Doi: $10.1200 / \mathrm{JCO} .2007 .15 .8253$.

39. Mackillop WJ, Stewart WE, Ginsburg AD, Stewart SS. Cancer patients' perceptions of their disease and its treatment. Br J Cancer 1988;58: 355.

40. Mende M, Trautmann K, Rentsch A, Hornemann B, Schuler US, Ehninger G, et al. Patients' perspectives on palliative chemotherapy of colorectal and non-colorectal cancer: A prospective study in a chemotherapy-experienced population. BMC Cancer 2013;13:66. Doi: 10.1186/1471-2407-13-66.

41. Sapir R, Catane R, Kaufman B, Isacson R, Segal A, Wein S, et al. Cancer patient expectations of and communication with oncologists and oncology nurses: The experience of an integrated oncology and palliative care service. Support Care Cancer 2000;8:458-63.

42. Ford S, Fallowfield L, Lewis S. Doctor-patient interactions in oncology. Soc Sci Med 1996;42:1511-9. Doi:10.1016/0277-9536(95)00265-0

43. Hak T, Koëter G, van der Wal G. Collusion in doctor-patient communication about imminent death: An ethnographic study. BMJ 2000;321:1376-81. Doi: 10.1136/ bmj.321.7273.1376

44. Noguera A, Yennurajalingam S, Torres-Vigil I, Parsons HA, Duarte ER, Palma A, et al. Decisional control preferences, disclosure of information preferences, and satisfaction among Hispanic patients with advanced cancer. J Pain Symptom Manage 2014;47:5:896905. Doi: 10.1016/j.jpainsymman.2013.06.010 